\title{
A perspective on materials characterization for technology advancement and industrial growth: Emphasis on non-destructive evaluation
}

\author{
BALDEV RAJ \\ Metallurgy and Materials Group, Indira Gandhi Centre for Atomic Research, Kalpakkam \\ 603102 , India

\begin{abstract}
Materials are the index of human progress. The evolution of mankind is linked to materials, for example, stone age, bronze age, iron age etc. The success of use of materials towards better quality of human life depends on the capabilities for characterization of these materials, which leads to ensuring reliable performance of the materials and development of new, and more efficient materials. The materials characterization encompasses determining chemical constituents, microstructural characterization down to a few Angstroms level and defect assessment from a few micrometers to few millimeters in a wide variety of materials. Recent progress made in the nondestructive characterization of materials and the future trends and direction are discussed in this paper.
\end{abstract}

Keywords. Materials characterization; nondestructive methodologies; defects; microstructure stresses; structural integrity.

\section{Introduction}

Materials are the index of human progress. Indeed, the evolution of mankind has been linked to materials and metals, for example stone age, bronze age, iron age, etc. Man's endeavours through evolutionary periods and civilizations have been possible due to development of appropriate materials and associated shaping technologies. We have come a long way from use of metals and alloys to increasing use of man-made composites and promising applications of structural ceramics. The present era of development is indicative of the needs to look at the materials with a global perspective. This means that use of metals and alloys, composites and ceramics should be dictated by their performance capabilities and economic viabilities. Various categories of materials are complementary, rather than competitive, in our endeavours to achieve better quality of human life. The successful use of materials demands adequate characterization of materials. Materials characterization is a front line activity of significant excitement for research and has application value. The need for chemical characterization of materials, microstructural evaluation at a few angstrom levels and defects assessment from a few micrometers to a few millimeters in a wide variety of materials and components has given rise to a tremendous range of techniques and instrumentation. The rapid pace of progress in computation, automation and visualization has helped the field of materials characterization to achieve the objectives of correlating characterization parameters with performance behaviours of materials in a meaningful way.

Materials science and engineering is a multi-disciplinary activity with abundant excitement and applications. Indians working with metals in earlier eras took pride in

Text of the Presidential Address delivered at the Materials Science Section of the 82nd Indian Science

Congress held at Calcutta during January 3-8, 1995. 
combining objectivity with quality and aesthetics. Ornamental 'WOOTZ' swords and bronze guns are a few examples. India, after independence, has strived to keep the traditional excellence in materials though the pace, many a times, has not matched with the developed countries. This can be due to many reasons including social necessities of the nation, motivation and science management. I would rather not like to go into the details of this analysis. However, $I$, on the basis of my association with the discipline of materials science and engineering for the last twenty-five years can say with conviction and pride that the foundation of materials science is excellent in the country. Indeed, in a few select areas of materials science, where we took the initiatives along with the rest of the developed world, we have made distinct impressions. This would not have been possible without the excellence in materials sciences, sound metallurgical education and maturity of research teams and industrial groups. A few examples in this category are extraction, refining and forming of rare metals and alloys including metallurgy of plutonium metal, its alloys and compounds, fullerenes, rapidly solidified alloys, superconducting materials, non-destructive evaluation etc.

Materials characterization is an interdisciplinary area where complementary contributions of chemists, physicists, metallurgists and instrumentation specialists enable success in technology. Chemical analysis (mass spectrometry, spectroscopy, chromatography etc), microstructural evaluation (optical microscopy, transmission electron microscopy, field ion microscopy, scanning ion mass spectrometry, scanning electron microscopy, X-ray diffraction etc), mechanical properties evaluation (hardness, tensile, creep, fatigue, fracture toughness, impact, etc) and non-destructive evaluation (X-ray, ultrasonics, eddy currents, laser, magnetic methods, etc) play important and complementary roles in materials characterization. Thus, it becomes imperative that choice of techniques, instruments, measurement parameters and analysis methods is done in a judicious manner without losing sight of cost-effectiveness and performance requirements.

$I$ intend dealing with an interesting and exciting facet of characterization i.e. assessment of materials, components and plants with the help of non-destructive evaluation (NDE) techniques and measurements. The topic is a natural choice for me as I have been actively involved in this area of science and technology at national and global levels. Also I have shared the pangs of pain and excitements in the growth of this interdisciplinary area of research, development and applications. It has so happened that when I started working in the area of NDE (in 1970), this discipline was in the process of transformation from heuristics and skill based technology to the science based technology. The strength of many NDE techniques which derive their origin from sound physical principles were being explored in 70 s and $80 \mathrm{~s}$. During those decades, basic studies using these techniques have led to unfolding the mysteries of materials and their behaviour thus attracting many academicians and researchers to the fold. It has been the most rewarding experience for me to grow along with this activity. The confidence of this experience gives me courage to look forward (with great hopes) to the future where NDE would make increasingly important contributions to realize new and better materials capable of performing with high reliabilities in demanding applications.

Non-destructive evaluation enables assessment of microstructures, defects and stress state in materials, components and plants without in any way impairing their usefulness for the intended performance and application. The field of NDE in medical practice is related to diagnosis. The doctors utilize techniques like X-ray tomography, 
nuclear magnetic resonance imaging, ultrasonic tomography etc for diagnosis and recommend measures to recover from inadequacies. NDE measurements in engineering practice provide information to help avoid failures, assess the condition for preventive maintenance and generate data for damage assessment and life prediction. While the purpose of medical diagnosis is to help to cure and to provide relief and comfort, the purpose of engineering NDE measurements is to enhance productivity, economy and safety. Thus NDE science and technology leads to realization of laudable objectives i.e. sound human beings operating sound engineering components and plants.

NDE applications that you would not normally hear (but equally important) are counting holes in a swiss cheese without cutting it up, locating knots in logs before starting to saw or checking porks and beans to pick out foreign objects inadvertantly carried during a production line accident. Another less known but vital application of NDE includes characterization, restoration and expert appraisal of works of art (paintings, icons, etc).

NDE of composites poses problems due to anisotropy. Methods have been developed based on macro and micro ultrasonic scanning, low energy tomography, digital X-ray imaging and millimeter range microwave techniques. Success lies in circumventing noise by adaptation and development of novel signal analysis and imaging approaches.

NDE of structural ceramics requires very stringent characterization of surface, sub-surface and volume defects. The size of volume defects needs to be resolved and evaluated, is in the range of $15-30 \mu \mathrm{m}$. Microtomography, acoustic microscopy and laser scattering are some of the techniques which have shown the matching capability for high technology applications of these materials.

Now, I would discuss in details, some of the highlights of the work carried out in India in the area of NDE for components and plants with specific reference to metallic materials. I have drawn the examples from the research studies which have resulted in new findings and applications, where indigenous instruments, software and expertise have been harnessed to solve problems related to strategic technologies or where significant financial gains have been realized for the country by avoiding costly imports, shut-downs or accidents.

\section{NDE for assessment of structural integrity}

Periodic assessment of integrity of engineering components, structures and plants is assuming importance for both economic and safety reasons. It is now widely accepted that all components and structures possess 'anomalies', however smaller they are, and anomaly assessment is the sole way to control structural integrity reliably. In my view, anomalies are either defects or microstructural variations or stress variations which when not removed or repaired might lead to cause ultimate loss of integrity of the component or structure. It is well known that the locations where stresses and microstructural degradations exist are the potential sites for origin of defects. In the pre-service scenario, anomalies may be present in the raw material stage or might have been introduced during machining, heat treatment and assembling. Similarly, they may be generated due to deterioration of the material/component/structure as a result of one or combination of the operating conditions like temperature, stress, chemical and radiation environment. The damage can be due to creep, fatigue, stress corrosion, 
embrittlement etc. The damage mechanisms result in deterioration of mechanical properties, crack initiation and propagation, leaks in pressurized components and catastrophic failures. Hence, reliable performance of a component, structure or a plant depends on both pre-service quality and in service degradation of the component. The role of NDE in avoiding premature failure of the components/structures is ever increasing. NDE achieves this challenging task by way of detection and characterization of anomalies in materials. The NDE information on the above anomalies together with design parameters is taken into consideration for evaluation of integrity and assessment of life of the components/structures. Moreover, by knowing the growth rate of defects, it is possible to estimate remaining life of the component/structure/plant.

\section{Advances in ultrasonic inspection of welds}

Among all NDE techniques, ultrasonic testing (UT) methods are widely used for detection and characterization of defects in critical and important structural welds. One such application is the inspection of weld joints of thin walled fuel elements of pressurized heavy water reactors (PHWR). Uranium oxide fuel pallets are encapsulated in zircaloy- 2 cladding tubes of $0.37 \mathrm{~mm}$ thickness and sealed with end caps by resistance welding. Structural integrity of these welds assumes great importance in view of its critical nature. Till recently, helium leak test and metallography were the methods employed to ensure the integrity of these weld joints. These techniques have their own limitations. Helium leak test detects only those defects which are 'through and through' and metallography, being a destructive test, can not be carried out on all the joints. The quality assurance procedure did not give enough confidence when the target was almost zero failure rate to keep the coolant in the nuclear reactor as less radioactive as possible. For this challenging problem, a solution has been found by developing ultrasonic testing aided by digital signal analysis. The UT of the weld region is based on angle beam with shear wave using immersion pulse echo method. In view of the difficulties observed during this method, due to interference from geometric reflections from the upsets produced during welding process, easily implementable advanced signal analysis approaches have been used. These procedures result in reliable and automated detection of defects down to $5 \%$ wall thickness in the end cap weld joints.

Maraging steels are widely used in space industry for fabrication of rocket motor casings. To achieve the desired payload capacity, stringent quality control procedures to detect and eliminate even very tight fatigue cracks in maraging steel as well as its weldments are necessary and development of such procedures assumes a great importance. Though conventional UT pulse-echo technique is applied successfully for defect detection in wrought materials, the weldments (more than $6 \mathrm{~mm}$ thickness) pose problems to achieve inspection specifications. The upper mid-section of the maraging steel weldment is acoustically noisy as this region has a coarse and more textured microstructure. This leads to only a small amplitude difference in echo signal from a defect and that from noise. Very tight cracks produced in these welds by fatigue loading (simulating the cracks introduced during welding) were detected using both cluster and pattern analysis of UT data. Undoubtedly, implementation of this methodology for detection of such small defects greatly enhances the payload capacity of the rocket motors.

Performing NDE on austenitic stainless steel (AUSS) welds and castings is difficult due to: (i) severe attenuation at high frequencies, (ii) presence of anisotropic grain 
structure (dendrites) which cause the ultrasonic beam to bend (beam skewing), (iii) noisy behaviour of the material masking the pulses from defects and (iv) variations in material properties all along. In view of the above difficulties, even well recognized international standards have set the allowable defect size to be around $10 \%$ of wall thickness. It is well known that AUSS components and structures find extensive applications in various industries and they enjoy a dominant position (75\% approximately) among other stainless steels (this is chiefly because of high level of the fabricability and corrosion resistance for this class of steels). In this regard, there has been a continuous demand for the development of a reliable NDE methodology for defect detection in AUSS. Knowledge and databases pertaining to the above mentioned difficulties are very large, but the expertise to assimilate and apply is scarce. Intelligent and timely application of this knowledge is the key to successful NDE of AUSS welds. The expertise available at our Centre in the area of UT, advanced signals analysis and artificial intelligence have been effectively utilized to solve this problem. For the first time in India, an integrated prototype rule based expert system, which helps in taking quick and reliable decisions on the UT procedures to be adopted for efficient NDE of AUSS welds has been developed. The system considers the following details before making decision: dendritic microstructure of the weld, weld dimensions, geometry, joint type, welding procedure, metallurgical history, reflective characteristics of various types of defects, equipment parameters etc. Using this system, it would be possible to detect defects down to $2 \%$ wall thickness. Inspection of inaccessible regions and complex geometries would become simpler if ultrasonic beam path is known. Visualization of software for simulating the path taken by the acoustic/ultrasonic wave inside the weld is being realized as part of the expert system.

Fibre-reinforced composite materials are being increasingly used in defence, space and commercial applications due to their light-weight, high-strength and stiffness. For the Light Combat Aircrafts being developed in India, composite materials are extensively used. This necessitates the development and standardization of NDE techniques for meeting the stringent quality requirements of composite materials. Unlike metals and alloys, composites are heterogeneous in nature which make NDE challenging. Some of the NDE methods used for inspection of composites include, ultrasonic testing, acoustic emission, thermography, low energy $\mathrm{X}$-radiography and micro radiography, tomography etc. Among all. UT and thermography enjoy the status of being reliable methods for evaluation of composite structures. Different kinds of defects that can occur in fibre-reinforced composite plates during its fabrication and in-service are contaminants, voids, unpolymerized resin, resin rich and resin-starved areas, ply gaps, foreign inclusions, delaminations, crazing and surface scratches etc. Though attempts have already been made to detect and characterize defects using dedicated imaging system, a few less expensive alternate methods have been developed. These methods use conventional UT in conjunction with advanced signal analysis approaches, based on two different experimental methods viz. ultrasonic immersion and dry couplant.

\section{NDE of steam turbine blades}

Based on my experience, I have a strong feeling that it is not always necessary to employ advanced NDT techniques for the inspection of components. At times, the conventional techniques can be efficiently used. In this line, detailed procedures based on wet 
fluorescent magnetic particle inspection (MPI) techniques have been successfully developed for the evaluation of $235 \mathrm{MW}$ low pressure turbine rotors of the PHWRs. This effort consisted of performing MPI on turbine blades of all five stages and fir-tree root regions of the discs of all the stages adopting cable and yoke methods of magnetization respectively. These procedures have also been effectively used to perform MPI on the 5th stage blades in situ without lifting the rotor from the turbine bed. Defects detected by these procedures were reconfirmed and characterized using direct current potential drop technique. Detailed in situ metallography and residual stress measurements (using X-ray diffraction technique) were carried out to supplement the MPI observations and to assess the damage at those regions.

\section{NDE of aircraft landing gears}

The Country's defence requirements demanding highest level of quality is indeed a priority. The Centre is immensely contributing for various defence organizations in solving their quality control and inspection problems. The Centre is recently engaged in one such important assignment. The landing gear is one of the vital components in a fighter aircraft. The landing gear undergoes fatigue damage due to stresses generated during landing. Life of such landing gears depends on the number of landings in addition to any force landings. It is essential to estimate the accumulated fatigue damage before any crack initiation and also detection of subcritical cracks to avoid catastrophe. The Centre has developed various procedures for detection of fatigue cracks using high sensitive magnetic particle technique. The procedures developed can be adopted for inspection of landing gears either in situ condition or in dismantled condition. Besides MPI, use of UT was envisaged on the landing gears. Advanced UT signal analysis techniques could detect $2 \%$ WT defects without any ambiguity. This achievement is considered to be another hallmark of out endeavours in par or exceeding international levels. To extend life of the landing gears, it is essential to estimate the accumulated damage and predict the life of the landing gear. At present, the Centre is engaged in developing the methodologies for quantitative estimation of damage using X-ray diffraction based residual stress measurements, hardness measurements and in situ metallography.

\section{Advances in radiography}

Recent advances in X-ray tube technology have resulted in focal spots, which are an order of magnitude smaller, thus, giving rise to a whole new classification of microfocus radiography techniques. It is now possible to detect minute defects in thin components, thin welds of steel, aluminum or titanium alloys, which were earlier not possible by conventional radiography. The most impressive feature of the microfocus $\mathrm{X}$-ray source is its ability to produce extremely sharp radiographs at exceedingly short film to focus distances. An outstanding example that brings out this capability is the work carried out on the radiography of tube to tube sheet welds in $2.25 \mathrm{Cr}-1 \mathrm{Mo}$ steel tubes of steam generators of prototype fast breeder reactor (PFBR). Inspection using conventional radiography is not possible due to limited resolution and problems of accessibility. The possible choice of using advanced microfocal radiography technique appears promising. Experience gained in this technique has been utilized to solve this problem, and 
achieved a sensitivity better than $1 \%$ wall thickness. Radiography techniques have also been employed for the NDT of carbon composite panels which are of interest to aircraft industry. Projective magnification and image enhancement techniques have been applied to extract information from low contrast radiographs. It is a common experience that many a times radiographs are not taken properly, needing to repeat the work for optimizing the sensitivity. This results in revenue loss due to costly radiographic films. Based on the experience gained in the last two decades, a comprehensive expert system has been developed. This expert system ensures a good radiograph to be taken without any possibility of mistakes, by the personnel, by identifying the radiographic conditions to be employed for a given application. More recently, radiography techniques have been successfully applied to detect any discontinuities in the electrical conductivity due to the presence of small porosities and other defects in a small diameter superconducting wires which are fabricated indigenously.

\section{Advances in acoustic emission technique for materials research and structural integrity}

Many components in high performance structures are fabricated from structural alloys which, while having high strength levels, have relatively poor crack arresting capability. Small cracks which have a greater potential to remain undetected, can approach critical size with very little extension. For these types of structures, only AET offers reliable capability to detect any increment of crack growth during structural loading as it can detect and assess growing cracks down to a size of $25 \mu$, even in the presence of noisy environments. Further, AET is very useful in predicting fatigue failures in advance. AET has been successfully applied to detection and monitoring of corrosion fatigue, hydrogen embrittlement and stress corrosion cracking. Pressurized pipes such as gas pipe lines and heat transport piping in power stations, are generally designed to satisfy the leak before break concept (i.e. a crack in a tube grows to cause a small leak long before reaching the critical crack size for catastrophic pipe rupture). However, this concept requires that a leak be detected at an early stage. Thus, AE monitoring is a potential technique for continuous surveillance for detection of cracks/leaks. Due emphasis has been given in our Centre on the use of AET for the structural integrity monitoring applications and materials research applications. The studies on materials research helps in understanding the $\mathrm{AE}$ behaviour under different deformation processes, thus, enabling better interpretation of AE data obtained during structural integrity monitoring applications. AET has been successfully applied for understanding deformation and fracture process in austenitic alloys (AISI type 316 stainless steel and Nimonic alloy PE-16), oxidation behaviour in ferritic steels, glass-crystalline transition temperature determination and structural relaxation studies in metallic glasses and martensite transformation in unstable austenite (AISI type 304 stainless steel). In Nimonic PE-16 alloy, various AE parameters have been used to correlate the $\mathrm{AE}$ behaviour with particle shearing and Orowan looping processes taking place in the presence of gamma prime and decohesion and fracture of MC occurring during tensile deformation. AET has been used to study the martensite formation in cold worked AISI type 304 stainless steel during cooling after ageing and to determine the temperature range for its formation during cooling. The studies also firmly established that the martensite formation takes place by shear process. 
The expertise gained in the Centre on application of acoustic emission technique (AET) for detection of leaks remotely emerged as a timely solution when a crisis occurred in the form of a leak in the end shield system of the Rajasthan Atomic Power Station, Kota. The leak had developed in an inaccessible region. It was not only necessary to locate the leak to carry out the repair, but it was also necessary to detect presence of any other leak, and to locate the same. The expertise available in the Centre on the use of AET for structural integrity and application of advanced signal analysis concepts could be effectively utilized for solving this problem. Similarly, in another challenging campaign, we had applied AET for detection of a leak at an inaccessible region in one of the 306 pressure tubes of the PHWR located at the Madras Atomic Power Station. Moreover, our expertise could be extended to Gujarat State Fertilizer Corporation, Baroda for finding the structural integrity of carbon dioxide absorber vessel thus saving crores of rupees for the plant by avoiding costly shutdown and replacement of the absorber.

The AE signals generated in austenitic stainless steel are generally weak. An innovative approach has been adopted to amplify these weak AE signals. The technique involves simultaneous external injection of ultrasonic waves of selected frequency, which interact optimally with subcritical AE sources, giving rise to enhanced $\mathrm{AE}$ signals which otherwise would not have been released at those stress levels. Amplification factors of 2 to 20 could be obtained by employing different amplitudes of injected ultrasonic signals.

For the overall assessment of integrity of components, identification and characterization of localized regions subjected to micro and macro yielding is very essential. In this direction, pioneering work has been carried out in our Centre. AET has been successfully used to detect the microplastic yielding occurring during macroscopic elastic deformation in austenitic stainless steel (AISI 316). Also, studies unequivocally established the necessity of using an $\mathrm{AE}$ sensor with a resonant frequency close to the frequency of the AE signal (generated by the source at a given strain), in order to detect even a weak source with adequate sensitivity. A theoretical model has been developed to predict the approximate frequency range of the AE generated from dislocation sources operating during pre-yield and near yield tensile deformation. The frequency of the AE signal has been predicted from the event life time of the Frank-Read and grain boundary source operations in AISI 316 steel and the same has been verified experimentally. The above studies are directly useful in maximizing the sensitivity for $\mathrm{AE}$ detection and better understanding of the $\mathrm{AE}$ behaviour, thus paving the way for complete characterization of not only the crack initiation and growth but also the deformation including yielding taking place prior to crack formation.

\section{Advances in eddy current testing}

Tubes made of ferritic steels are used in many heat exchangers and steam generators in power, petrochemical and fertilizer industries. Detection of defects and wall thickness reduction in these tubes in-service is very important, particularly as the plants are reaching their design life. Steep increase in infrastructural costs has made it mandatory to use the existing plants to their fullest life, without compromising safety and productivity. Hence due thinking and attention is given to life estimation and life extension programmes. Presently, only ultrasonic testing is possible with limited 
success for the inspection of ferritic tubes. However, the higher speed of inspection achievable by ECT tempts one to adopt it. Though application of ECT for nonferromagnetic materials is a well established practice, the same is not the case with ferromagnetic materials because of the domination of spatial variation in the permeability on impedance change. The recent solution to this problem is the use of remote field eddy current technique (RFECT). Though application of this technique with encouraging results has been reported in literature and are considered highly valuable due to economic advantages accruable due to this technology, the details on the technique are held confidential. In a challenging project undertaken by the Centre, an indigenous RFECT system has been developed for the first time in India. Apart from this, the RFECT phenomenon has been theoretically modeled to understand the electromagnetic interactions, to predict the optimized test parameters for achieving enhanced defect detection sensitivity and finally to provide inputs to the development of RFECT system. The utility has demonstrated sensitivity of 10 to $15 \%$ wall thickness loss as compared to the internationally reported value of more than $20 \%$ wall thickness loss.

Expansion transition zones and rolled joint regions in heat exchangers are the potential sites for failures. Inspection of these regions for defects is a challenging and interesting problem. Though UT was used with limited success, the problem still remained unsolved till recently. One recent technique that has shown tremendous potential in detecting defects in these regions with equal sensitivity to both longitudinal and transverse defects is phased array ECT (PAECT). This system uses a substantially different bridge circuit and probes that produce a constant magnitude rotating magnetic field which is insensitive to expansion transition zone, tube sheet or support plate. With the expertise available at our Centre, a new tandem PAECT probe has been designed and developed. This probe minimizes the insensitive zones prevailing in the commercially available probes. This probe has successfully demonstrated enhanced sensitivity for inspection of tubes. This is first of its kind developed anywhere in the world.

In the pressurized heavy-water-reactors, which are the backbone of our nuclear industry, an important life limiting factor for the coolant channel system is the location of garter springs that are placed to separate the calandria tube operating at low temperature and the pressure tube operating at high temperature. During installation, commissioning or operation, there is a possibility for the shift in the garter springs. This shifting leads to sagging of the pressure tube with the consequence of contact between the pressure tube and the calandria tube. Contact between the two tubes may result in the failure of the pressure tube with serious consequences. Detection and location of garter springs is possible by ECT. The technology for ECT for detection of garter springs was neither available for buying nor any literature existed on the technology at the time of development. The development of ECT procedure needed expertise in design and development of suitable probes. Our Centre had taken up the challenge and using the FEM based design, the probe parameters were optimized and suitable probes were developed. These probes have been successfully utilized for detection and location of garter springs in PHWR. The probes necessary for yet another important application i.e. estimation of gap between the calandria and pressure tubes, have also been designed and fabricated.

NDT has benefitted with the availability of relatively low cost Personal Computers and the accompanying revolution in digital technology. A recent outcome of these advances in the area of ECT is eddy current imaging (ECI). For the first time in India, in par with the internationally available systems, a computer based ECI system has been 
developed in our Centre to scan the object surface and to produce 2-D impedance images of defects. Imaging greatly enhances the defect detection capability. Fatigue cracks, corrosion damage and other surface and subsurface anomalies can be imaged and characterized. ECI has been used to detect weld centre line in stainless steel welds with an accuracy of $0.1 \mathrm{~mm}$. This would enable positioning of other NDE sensors. ECI is finding wide applications in aircraft industry.

\section{Holographic NDE}

Holography is becoming more and more popular in NDE as its capabilities are being increasingly understood. Holographic interferometry (HI) technique, being non-contact, is finding a place as a complementary or sometimes unique NDE technique in many specialized applications. It is being routinely used in aircraft industry for inspection of aircraft tyres, microwave antenna etc. This method has shown promising results in the NDE of composite panels, honeycomb structures and bond assessment in adhesively-bonded structures. Significant expertise has been gained by the group in this area. HI has been shown to be an useful tool to investigate the deformation states on the surfaces of pressure vessels. The defects in such components could be differentiated on the basis of their severity. Fringe pattern density is used to find the nature of defects (symmetric or asymmetric etc). Fabrication and welding processes alter the mechanical properties of the material and introduce residual stresses leading to manifestation of non-uniform deformations. HI technique has been used to measure these deformations, establish fitness-fer-purpose and suggest changes in the manufacturing process and design. Holographic NDT is particularly useful on complex shapes where other NDE methods such as UT and RT are difficult or time consuming to apply. In another application, for precise measurement and characterization of variations in diameter of the order of a few microns that occur during irradiation of the nuclear fuel cladding tubes (stainless steel), holographic interferometry techniques have been used.

\section{Laser scattering for inspection of heart valves}

Laser scattering techniques, being non-contact are also widely used for a variety of applications. I would like to share one recent important application of this technique for surface roughness measurement of heart valve discs. UHMW-PE artificial heart valves are being developed indigenously by Sree Chitra Tirunal Medical Institute, Thiruvananthapuram. Surface finish plays an important role on the bio-compatibility for human implantation. Conventional methods like stylus-profilometry can damage the valve surface. The possible choice of using laser scattering technique appears promising. Expertise gained in this area has been utilized to solve this problem and established a good correlation between surface finish and laser scattering parameters.

\section{Thermography for electrical installations}

Another area is the application of thermography for inspection of electrical installations. Many a times there are disruptions in the power supply due to failures in the components of the electrical installations. One of the main reasons for this is the 
formation of hot spots in the components due to loose contacts and accumulation of dust, etc. It is recommended that timely detection of these hot spots and their rectification would avoid shutdowns and interruptions in the power supply. The most versatile technique is the thermography, which would map the temperature distribution in a component thus enabling detection of hot spots. Periodic inspection of electrical installations has helped in formulating preventive maintenance and efficient functioning of the electrical installations.

\section{NDE for on-line monitoring of welding}

The present day automatic welding systems do not have the adaptive features of welding process control unlike manual welding wherein the welder alters the welding parameters utilizing his skill and experience and depending on his visual observations during welding. An approach to the adaptable and automatic welding system lies in the development of on-line sensing techniques capable of giving precise information about the origin and growth of defects. Two well-established techniques in this context are thermography and acoustic emission. These techniques give real time information regarding the weld quality and defect formation thus offering on-line rectification/ modification of welding procedure resulting in elimination of defects, reduced scrap and repairs towards visualizing the concept of 'intelligent processing of materials'. In addition, this would also bring down the need for carrying out post-weld NDT. In my opinion, on-line techniques are particularly useful and cost effective for high technology areas like nuclear, aerospace etc where welds with stringent specification for defects acceptance are a must for higher reliability. Event rate and RMS voltage of AE signals could be used to detect, locate and differentiate weld cracking, inclusions and process and equipment malfunctions like short circuit, current variations and arc drifting etc. The adaptability of thermography has been successfully established through systematic studies by our scientists. A variety of defects viz. porosities, lack of side wall fusion, lack of root penetration, undercut and blow holes were introduced during the welding process (metal arc and gas tungsten arc welding) and the thermal profiles were recorded and analysed to identify the presence and nature of defects. Studies have firmly established that the technique can pave the way for producing reliable welds in a most cost effective manner.

\section{NDE methodologies for microstructural characterization}

The knowledge of microstructural variations in a component is also very important, besides defect details, to ensure the desired performance. This is because the locations of microstructural degradations and stresses are the potential sites for origin of defects. Traditionally, microstructures are investigated by microscopy, which are destructive in nature. Thus there was an immediate necessity for the development of NDE techniques, for detection and characterization of microstructural variations. In this context, pioneering work carried out by our scientists established the adaptability of conventional and new NDE techniques for investigation of microstructural degradations. In some situations, advanced concepts have been augmented with the conventional NDT techniques. All these methodologies have been successfully found to be useful for damage assessment, life estimation and extension applications of ageing plants and 
components, in general, and for assessment of the suitability for continued service of components subjected to unacceptable service conditions during system malfunction or under accidental conditions, in particular.

Ultrasonic velocity and attenuation measurements have been successfully carried out on important metallic materials viz. Zircalloy-2, $9 \mathrm{Cr}-1 \mathrm{Mo}$ steel, $2 \cdot 25 \mathrm{Cr}-1 \mathrm{Mo}$ steel, 17-4 PH steel, Nimonic alloy PE 16, A533B pressure vessel steel, aluminum alloy weldments, carbon steel and stainless steels towards characterization of microstructural variations. Some important applications in this context include (i) characterization of precipitation behaviour and estimation of volume fracture in Nimonic alloy PD 16, (ii) assessment of microstructural degradation (dissolution of tempered martensite structure to ferrite and carbides) in $9 \mathrm{Cr}-1 \mathrm{Mo}$ steel, (iii) characterization of precipitation hardening behaviour in $17-4 \mathrm{PH}$ steel, (iv) characterization of various microstructures in A533B pressure vessel steel, (v) estimation of porosity in aluminium alloy weldments, (vi) determination of texture coefficients in cold worked 304 stainless steel and (vii) assessment of creep damage in low alloy ferritic steels. In view of the difficulties noticed during routine procedures for characterization of microstructures in Zircalloy-2, advanced neural network approach has been used and found to be very efficient in identifying the presence of various phases. Similarly, microstructural characterization in highly attenuating materials such as concrete, stainless steels and rocks is rather difficult by attenuation methods. Besides, the change in velocity in such components is expected to be of the order of $0.1 \%$. To meet this requirement, in our Centre, a methodology has been developed for precise ultrasonic velocity measurements using cross-correlation technique. The accuracy in the velocity measurements is enhanced by adopting cubic spline fitting of the cross-correlation function. This methodology has been applied for finding the influence of microstructure on ultrasonic velocity in $9 \mathrm{Cr}-1 \mathrm{Mo}$ ferritic steel and 17-4 PH stainless steel. Many of the results established in the area of microstructural characterization, would be directly useful in developing procedures for microstructural degradation, damage assessment, life extension applications in addition to quality control during fabrication and heat treatment.

Besides UT, magnetic methods are the potential methods for evaluation of microstructural degradations. Magnetic flux perturbation and acoustic emissions are generated when an induced magnetic field in ferromagnetic materials is swept in a hysteresis loop. The former is referred to as magnetic Barkhausen noise (MBN) and the latter is called acoustic Barkhausen noise (ABN). Various microstructural features such as grain boundaries, precipitates, stresses etc affect the nucleation and movement of magnetic domain walls. Hence, MBN technique is finding applications in several areas including microstructural characterization, characterization of post weld heat treatment in the weld joints and the measurement of residual stresses. Significant work has been done by our scientists in this direction. In the case of 17-4 PH steel, MBN technique has been employed to identify the stages i.e. (i) build up of coherency strains, (ii) loss of coherency and (iii) interlath austenitic formation which otherwise need transmission electron microscopy and field ion microscopy investigations. In the case of $2 \frac{1}{4} \mathrm{Cr}-1 \mathrm{Mo}$ steel, precipitation of $\mathrm{Mo}_{2} \mathrm{C}$ could be identified using $\mathrm{MBN}$ technique. Recrystallization from martensite structure leading to ferrite formation in addition to coarse carbide precipitation could also be characterized. In $9 \mathrm{Cr}-1 \mathrm{Mo}$ steel, the incipient occurrence of delta ferrite could be detected by magnetic techniques. By even transmission electron microscopy, it is difficult to identify the incipient occurrence of delta ferrite. MBN technique has been successfully used for the characterization of post 
weld heat treatment in the simulated weld joints of steam generator modules of the proposed PFBR. These studies efficiently brought out the adaptability of the MBN analysis for quality assurance of welds. ABN technique has been used for assessment of residual stresses in weld joints of structural steels. The RMS voltage of the ABN signal has been used as a parameter for studying the influence of stress relief annealing treatment on the stress distribution. Increase in RMS voltage of the ABN signal corresponding to decrease in the residual stress could be observed, after stress relief annealing. In the as welded condition, stresses are higher at the centre of the weld region and decrease gradually at the regions away from it. Reduction in stresses due to annealing could be identified in the form of increase in ABN signal. Utilization of $M B N$ and $\mathrm{ABN}$ data for assessment of microstructures is emerging as a very promising technique for controlling heat treatment and for damage detection and life prediction of components.

\section{NDE of residual stresses}

As I mentioned earlier, besides information on defects and microstructure, the knowledge of residual stresses in a component is also needed for performing reliable NDE. Residual stresses are the system of stresses which exist in a structural material or component when the material/component is free from external loads. They are generally caused by inhomogenous plastic strains in a structure. Manufacturing processes are the most common causes of residual stress. In some instances, residual stress may also be induced later in the life of the structure by fatigue, by occasional overloads, in-service repair or modification. While tensile residual stresses are generally detrimental, increasing the susceptibility of a component to failures by fatigue or stress corrosion cracking, compressive residual stresses are usually beneficial, as these stresses overcome the above drawbacks. In this area too, a remarkable progress has been made using NDE technique for detection and assessment of residual stresses leaving behind the obsolete destructive method, namely, hole drilling and dissection methods. Today, simple, rapid, automated and well established NDE techniques/procedures are available for residual stress measurements. Among others, the NDE techniques that have good potential include ultrasonic testing, magnetic Barkhausen noise analysis (MBN) and X-ray diffraction. It gives me immense pleasure to state that an MBN utility has been developed indigenously in our Centre which is extensively used for microstructural characterization and residual stress measurements. Residual stress measurements were carried out on many technologically important components using these techniques. $2 \cdot 25 \mathrm{Cr}-1 \mathrm{Mo}$ tube to tube sheet calibration welds of PFBR heat exchangers were studied to evaluate post weld heat treatment for stress relieving.

Explosive welds which have a wide variety of applications have been systematically analysed by XRD technique for finding stress distributions. This study has also revealed for the first time, the appearance of an X-ray peak due to formation of a new phase at the weld interface. Similarly, the effect of plate thickness on residual stress distribution in butt welds of another important structural steel has been established successfully. An important task performed recently includes the meticulous mapping of residual stresses in 316 stainless steel rounds used for fabrication of components for control rod drive mechanism of fast breeder test reactor. This work has elegantly optimized the heat treatment cycle towards bringing down the residual stress levels in 
the final components. Stainless steel dished ends, which are common for vessels used in various industries, are normally fabricated by cold pressing or cold spinning. This process gives rise to high level of residual stresses. These dished ends when put into service may fail by stress corrosion cracking. The Centre's expertise has been utilized to device a judicious combination of approaches involving NDE techniques for residual stress measurement, refined heat treatment cycle and modifications of alloy composition to avoid premature failures of the dished ends. Another pioneering study has established the potential of XRD studies for microstructural characterization in 17-4 PH steel. Studies confirmed that full width at half maximum and peak position of a specific crystallographic plane are better parameters to assess ageing of the steel as compared to presently practised hardness measurements which can not distinguish underaged/overaged conditions. This study has, undoubtedly, direct implications for in-service inspection.

\section{Future trends and directions}

The expertise and techniques developed in the Department are indeed colossal and can cater to national demands and challenges. However, the spread of expertise can happen mainly by lateral interactions, thus leading to absorbing the technologies by the needy organizations. The Department, on its own, is conscious of not loosing the lead and continue to work at the frontiers of research and development in the area of nondestructive testing and evaluation and exploit the same for various applications.

There has been a continuous and ever increasing demand for the NDE professionals for the development of new techniques and procedures to achieve 'minimum defect component with maximum confidence and reliability' goal for the structural integrity of critical components. Tremendous effort put globally to meet this challenge has yielded encouraging results. For pre-service inspection, a definite requirement exists for more accurate NDT techniques which could be applied with the aim of producing a structure with almost zero defects. Advancements in the fields of electronics, instrumentation and computer sciences, development of new methodologies, better understanding of interaction between the interrogating media and materials and new challenging requirements are pushing the NDE technology to visualize the above need. A major spin-off in the recent past in this direction is intelligent processing of materials'. This concept ensures $100 \%$ acceptability of raw materials and fabricated components by providing feedback to the manufacturing/fabrication processes about the presence/occurrence of defects on-line, thus allowing for immediate corrective action.

Scientific measurements based on a single sensor or probe can provide only limited information. The synergistic use of measurement data from a probe array comprising of multiple sensors will increase the analytical power of the NDT technology by providing complimentary information from different sensors. At present, NDT and inspection surveys represent a disparate collection of data from different sensors applied on an individual basis. A relatively new concept called 'Data Fusion' will combine the signals from a range of NDT sensors to create a global picture of the fitness for purpose of the inspected zones. Also it involves combining data from more than one NDT method and provides a comprehensive description of an anomaly. In otherwords, if the 'shadow' information from a radiograph is combined with depth information 
from an ultrasonic shear wave probe and surface breaking information from a magnetic particle inspection, then the NDE personnel can provide a comprehensive description of the defect. Data fusion makes it much easier for non-specialist to interpret the results of inspection in a reliable manner.

Similarly, imaging techniques show a great promise towards enhanced detection and characterization of defects in components. It has often been said that an NDE image is worth thousand NDE signals. Imaging can be done with many different media including optical, infrared, X-ray, gamma-ray, ultrasound, eddy current, thermal wave and magnetic resonance etc. Imaging format provides global perspective of the inspected region and allows a balanced interpretation. Also, imaging techniques have the potential of automating the measurement process, providing estimates of defect size from the image data, producing accurate characterization of defects and improving the probability of detection.

Technological advancements, that are evolving in the fields of science and technology that are apparently unconnected to NDE, are beginning to have a profound effect on NDE. Neural networks, pattern recognition, speech and signal processing, fibre optics, computers, robotics, knowledge base expert systems, fuzzy logic etc hold significant promise. Use of these nascent technologies and the synergistic merits of these modern fields as applied to NDE has resulted in enhanced sensitivity and reliable inspection methodologies. Development of an integrated prototype rule based expert system to be used as an advisor for ultrasonic testing of austenitic stainless steel welds, visualization software for simulating the path taken by an acoustic/ultrasonic wave inside a material and microstructural characterization using artificial neural networks are some of the major spin-offs in this direction. The use of robotic devices for reliable NDE of components is assuming great importance in many industries particularly nuclear, space and chemical especially when there is a need for high reliability for defect detection and limiting the operator dependency. Manipulators based on robot principles have come into existence and the demand for the manipulators with computerized data acquisition, processing and evaluation is growing faster for pre-service and in-service inspections.

Ever since India attained independence in 1947, there has been a sustained and comprehensive approach adopted for the development of industries for production of quality, precision, strategic and heavy components. To achieve consistent success in all the industrial ventures, the essential requirement is the unflinching commitment to quality through non-destructive testing. With increasing global competition, the demand on quality of products and services has increased many fold in recent times. Quality of products and services is essential due to product liability, government regulations, reliability, safety, economic benefits etc. During quality management, till recently, the concept of error (defect) detection rather than error (defect) prevention was adopted. For this, the key area of technology is the nondestructive testing. However, error prevention rather than detection is given preferential treatment in the modernday-world as zero based defect concept with lower costs and higher reliability is the ultimate goal. Quality of products can be achieved only when various aspects of quality are taken into account during different stages including design. material conformance, product performance and service. Additionally, quality concepts are also adopted during selection of materials, specifications which ensure quality, and to enhance reliability, maintainability and production. The quality of products can be achieved only when due attention is paid to design review, reliability analysis, review of drawings 
and documents, evaluation of the product, failure modes and analysis and corrective actions.

Nation has also realized that quality has to be built into the product for global competitiveness. This has led to the development of 'systems' related quality, i.e. 'process control related quality assurance' and 'new product oriented quality assurance' in addition to conventional 'inspection related quality assurance'. Statistical quality control (SQC) based on Juran approach and Crosby approach, total quality control based on Ishikawa approach are adopted to avoid $100 \%$ inspection, but at the same time, ensuring the quality of all the products. Taguchi approach consisting of product design and tolerance design techniques are also being applied. Quality is also developing links with standardization, value engineering, inventory control, factory automation, robotics for automatic inspection, CAD/CAM and market research. It is clear that the technology has a definite role and also the human attitude and cultural change from the top manager to the shop floor worker plays an important role in achieving total quality. Quality-Science and Technology is a multi and inter disciplinary topic encompassing the knowledge of design, materials selection, metallurgical aspects (microstructure and properties), materials specifications, production technology, maintenance, statistics, repair technology, failure analysis, NDT technology, automation, robotics, computers, information systems etc. While many NDE techniques and procedures are available, their adoption for quality in a judicious manner, taking into account the above given philosophy and approaches for 'quality' is the current effort in the light of global competition and economic liberalization.

NDT needs to be practiced.widely by the Indian industry to improve the quality of the products/services. Industry is conscious of the rich benefits that follow the implementation of strict Quality control, Quality management and Quality audit methodologies. Well-established and standardized NDT techniques/procedures should be regularly upgraded and adopted by the Indian industry. Research organizations attached to Department of Atomic Energy, Space and Defence and the premier educational institutions like the Indian Institutes of Technology and Indian Institute of Science perform the basic research and development in the field of advanced NDT. These organizations must make conscious and committed efforts to transfer the acquired and much needed know-how to the industry. Most of the important industries in India are required to upgrade their facilities and quality control and audit programs for obtaining the ISO 9000 accreditation in view of the liberalized policy of the government. In this regard, training of personnel with the state-of-the-art NDT knowledge through certification courses, national and international workshops, conferences and meetings assume utmost priority. When I examine the ISO- 9000 concepts, I find that the seeds of these concepts that are sweeping the industry today were sown in the nuclear and aerospace industry a few decades ago and being followed regularly since then. An important philosophy preached and adhered to by these industries is that cost-effectiveness and quality are inseparable partners for obtaining long-term benefits from any industrial endeavour of mankind. These objectives set the trends for significant progress in the science and technology of NDT towards achieving zero defect criterion. The stringent quality control procedures and methodologies followed by these industries can be adopted by power, petrochemical, transport and other industries as well, with significant advantages.

Alrein Weinberg, a pioneer in the evaluation of science gives two criteria for the assessment of the value of science: (i) The underlying value in the practice of science is 
truth and what achieves truth efficiently is the most valuable and (ii) the underlying value in the administration of science is utility and what is most useful (in addition to its being true) is the most valuable. Judged by these two criteria, the discipline of materials characterization in general and NDE in particular, satisfies the Alrein Weinberg criteria due to the intrinsic strengths of good material characterization work, be it related to research or application. I do not know of any other field which has achieved such a laudable objective: sound human beings operating sound engineering components and plants. The expertise in the field of NDE is extended further to characterization of earth (its interior) and environment for pollution and other changes. NDE, science and technology is contributing significantly to achieve better human life for our citizens and world citizens at large. The strengths built over four decades in India can be exploited and enhanced further to make India the international leader in the coming vears. 